\title{
Carbon Emissions and Economic Development in Transport Industry_An Empirical Research Based on Decoupling Theory and Structure Decomposition Model
}

\author{
ZHANG Shiqing $^{\mathrm{a}}$, ZHENG Wenlong ${ }^{\mathrm{b}}$, WANG Jianwei ${ }^{\mathrm{c}}$
}

School of Economics and Management, Chang'anUniversity, Xi'an 710064, China

a,czh_chd@163.com, ${ }^{\mathrm{b}}$ 534236243@qq.com

Keywords: transport sector; carbon emission; decoupling theory; influence factors; LMDI

\begin{abstract}
In order to study the relationship between transportation carbon emissions and economic growth, driving factors of carbon emissions, we established elastic decoupling model and LMDI decomposition analysis model of transport sector. Taking Beijing Tianjin Hebei region as an example, the empirical study was carried out.Results showed that Tianjin and Hebei were in weak decoupling state, Beijing was in expanded negative decoupling state. And the transportation output scale effect in Beijing, Tianjin and Hebei was the main factor to boost the growth of carbon emissions.Energy intensity effect has led to the reduction of carbon emissions in the transport sector in Tianjin and Hebei, but has led to the increase of transport carbon emissions in Beijing.In terms of the energy structure effect, the transportation energy structure effect of Beijing, Tianjin and Hebei have weak positive contributions or negative contribution to the overall of carbon emissions.
\end{abstract}

\section{Introduction}

Resolving the contradiction between economic growth and carbon emissions properly is an important task for all countries.The establishment of low carbon transportation system has an important effect on China's response to climate change and carbon emission reduction targets [1]. Therefore, it is urgent and significant to study the decoupling relationship between carbon emissions and economic growth and driving factors of carbon emissions in China's transportation industry.

Decoupling theory is used to measure the relationship between material consumption and economic growth in the process of industrial development. Tapio [1] put forward the theory of elasticity decoupling. The decoupling of carbon emissions and economic growth in the transportation industry was decoupled from the economic growth. Yang [2] used the Tapio elastic decoupling model to analyze the relationship between carbon emissions and economic growth in China's transportation industry.In terms of application and study of carbon emissions structure decomposition, Ma Yueyue et al [3] used LMDI decomposition method to analyze the influence factors of carbon emissions in China logistics industry.But little research focuses on decoupling relationship between carbon emissions and economic growth, and driving factors of carbon emission of transportation industry in Beijing, Tianjin and Hebei.

Beijing, Tianjin and Hebei region is one of core city circle, and also our country's political, cultural, scientific and technological center, whose transportation industry is also facing a huge energy saving and emission reduction challenges and pressure. Therefore, it is of great significance to carry out the empirical analysis on the carbon emissions decoupling effect and driving factors.

\section{Materials and Methodology}

In order to ensure the accuracy of carbon emissions accounting of the transportation, took 21 kinds of energy as the statistical object to calculate the carbon emissions (D) of the transport industry, see Equation (1).

$D=\sum A_{i} \times B_{i} \times C_{i} \times N_{i} \times \frac{44}{12}(1)$ 
D stands for carbon emissions of energy consumption in the transport industry; $A_{i}$ stands for the consumption of EnergyI; $B_{i}$ stands for standard coal coefficient of EnergyI; $C_{i}$ stands for carbon emission coefficient of I; $\mathrm{N}_{\mathrm{i}}$ stands for fuel oxidation rate of Energy $\mathrm{I}$.

The decoupling between carbon emissions and economic growth is carbon emissions are not increased with the growth of the economy, but even appear the decline trend.Based on the Tapio elastic decoupling model, the elasticity decoupling model of the relationship between transportation industry carbon emissions and economic growth wasput forward, as shown in the formula (2).

$$
t_{\mathrm{CO}_{2, I G D P}}=\left(\Delta \mathrm{CO}_{2} / \mathrm{CO}_{2}\right) /(\triangle I G D P / I G D P) \text { (2) }
$$

$\mathrm{T}_{\text {co2, IGDP }}$ is elasticity decoupling value of carbon emissions from transportation industry and transport industry GDP(One hundred million yuan);In order to eliminate the factor of price variation,transport industry's total production value was calculated with the constant price.

This paper used extended Kaya identity and Logarithmic Mean DivisiaIndex(LMDI) to study driving factors of carbon emission.

$$
C=\sum \frac{C_{i}}{E_{i}} \times \frac{E_{i}}{E} \times \frac{E}{I G D P} \times I G D P=\sum f_{i} \times s_{i} \times n \times g
$$

$C_{i}$ stands for the amount of carbon emissions from the consumption of Energy I; $E_{i}$ stands for the amount of consumption of Energy I; E stands for total energy consumption; IGDP stands for gross product of transport industry; $f_{i}=C_{i} / E_{i}$ respects for carbon intensity effect; $s_{i}=E_{i} / E$ respects for the proportion of Energy I in total energy and it is used to explain the structure of energy consumption; $\mathrm{n}=\mathrm{E} / \mathrm{IGDP}$ stands for energy consumption intensity; g stands for influence of industrial scale on carbon emissions in transportation industry

Using LMDI model to decompose formula (3), change of carbon emissions from the period to $\mathrm{t}+1$ period $(\triangle \mathrm{C}$ )in Beijing, Tianjin and Hebei region can be expressed as the formula (4).

$$
\begin{aligned}
\Delta C & =C_{t+1}-C_{t}=\Delta C_{f}+\Delta C_{s}+\Delta C_{n}+\Delta C_{g} \text { (4) } \\
\Delta C_{f} & =\sum \frac{C_{i}^{t+1}-C_{i}^{t}}{\ln C_{i}^{t+1}-\ln C_{i}^{t}} \times \ln \left(\frac{f_{i}(t+1)}{f_{i}(t)}\right) \text { (5) } \Delta C_{s}=\sum \frac{C_{i}^{t+1}-C_{i}^{t}}{\ln C_{i}^{t+1}-\ln C_{i}^{t}} \times \ln \left(\frac{s_{i}(t+1)}{s_{i}(t)}\right) \\
\Delta C_{n} & =\sum \frac{C_{i}^{t+1}-C_{i}^{t}}{\ln C_{i}^{t+1}-\ln C_{i}^{t}} \times \ln \left(\frac{n(t+1)}{n(t)}\right) \text { (7) } \Delta C_{g}=\sum \frac{C_{i}^{t+1}-C_{i}^{t}}{\ln C_{i}^{t+1}-\ln C_{i}^{t}} \times \ln \left(\frac{g(t+1)}{g(t)}\right)
\end{aligned}
$$

$\triangle C_{f}, \triangle C_{s} 、 \triangle C_{n} 、 \triangle C_{E}$ respectively stands forcontribution to the carbon emissions change value of carbon emission intensity effect in transportation industry, energy structure effect, energy consumption intensity effect, industrial scale effect. Because of all kinds of energy's carbon emissions coefficient remains constant, so $\triangle \mathrm{C}_{\mathrm{f}}=0$.

\section{Empirical Analyses}

Raw data are mainly derived from statistical yearbook of China and 2006 IPCC national greenhouse gas inventory guidelines, through Formula (1) to calculate the carbon emissions.Through Formula (2), the paper has calculated elastic decoupling of carbon emissions from transportation industry, and results were shown in Table 1.

Tab.1Average carbon emissions and of elasticity decoupling value from transport sector

\begin{tabular}{ccccccccc}
\hline Region & $C$ & $\Delta C$ & $\Delta C / C$ & $I G D P$ & $\Delta I G D P$ & $\Delta I G D P / I G D P$ & $T_{C, I G D P}$ & Decoupling State \\
\hline Beijing & 1611.56 & 146.06 & 0.110 & 513.07 & 33.50 & 0.068 & 1.754 & $\begin{array}{c}\text { Expanded negative } \\
\text { decoupling }\end{array}$ \\
Tianjin & 825.32 & 45.83 & 0.057 & 337.49 & 35.48 & 0.112 & 0.558 & Weak decoupling \\
Hebei & 1533.39 & 77.50 & 0.052 & 1100.71 & 119.41 & 0.119 & 0.426 & Weak decoupling \\
\hline
\end{tabular}

From Table 2 we can see average changes value of carbon emissions and industrial production value in 2005-2012 are positive number. It shows that carbon emission and industry gross domestic 
product present an increasing tendency. From the state of decoupling, the relationship between carbon emissions and economic growth in Beijing is in expanded negative decoupling state. It shows more efforts are needed to make in carbon emissions reduction. Tianjin and Hebei are both in weak decoupling state, showing carbon emissions growth rate is less than the growth rate of transportation economy. However, it has not realized strong decoupling. Compared with Beijing, effect of carbon emissions reduction in Tianjin and Hebei is better. Making more energy saving and emission reduction efforts is possible to achieve absolute carbon emissions.

Taking 2005 as the base period, trough Formula (3) (8) we calculated various influencing factors contribution of carbon emissions in Tianjin and Hebei region, seeFigure 1.
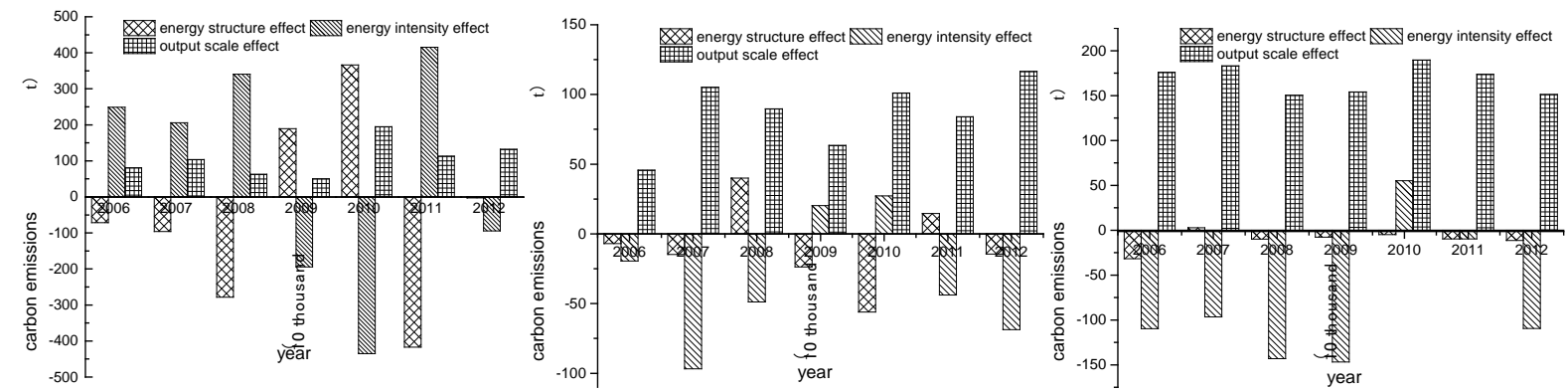

Fig.1Thevarious influencing factors contribution of carbon emissions in Beijing,Tianjin and Hebei

The transportation output scale effect leads to sharp increase in carbon emissions, and it is the main factor to boost the growth of carbon emissions. In terms of carbon emissions contribution effect of the output scale factors by time sequence, the output scale factor all maintain a higher level of contribution to the carbon emissions, and there is no apparent decline, indicating that the transportation economy growth is still based on energy consumption in Beijing, Tianjin and Hebei, relationship between economic growth and carbon emissions has not yet realized the ideal state of decoupling.

In terms of the factor of energy intensity, energy intensity effect has led to carbon emissions reduction in Tianjin and Hebei, and it shows the energy use efficiency is improved in these regions. The energy intensity effect leads increase of transport carbon emissions in Beijing, it shows that the transportation energy use efficiency is not high, the development pattern is extensive, the potential of energy saving and emission reduction is huge. In terms of the time sequence, in most years the energy intensity factors on carbon emissions have strong inhibition in Tianjin and Hebei, but the inhibition has decreased in recent years. Butthe energy intensity actor had a significant role in promoting transport carbon emissions in Beijing

In terms of the energy structure effect, the transportation energy structure effect of Beijing, Tianjin and Hebei have weak positive contributions to the overall of carbon emissions, and it suggests that transport industry of China's energy structure is still relatively single, it still is the traditional fuel such as diesel, gasoline, kerosene, coal. Although the proportion of new clean energy in the energy structure has been promoted in China, there is still little change in the original single energy structure. In terms of carbon emissions contribution effect of the energy structure factors by time sequence, the quantity of negative valve of energy structure factors are five years in Beijing and Tianjin, and the quantity of positive value are two years, and it shows that despite energy structure factor has a weak negative effect on carbon emissions in Beijing and Tianjin, but the effect of the adjustment of energy structure is not stable. The inhibitory effect was relatively stable in Hebei, but it was very weak, the adjustment of energy structure still needs to be optimized.

\section{Conclusions}

Tianjin and Hebei are in weak decoupling state, relationship between transport carbon emissions and industry economy development in Beijing is in expanded negative decoupling state.It shows that greater efforts are still needed to make in terms of carbon emission reduction in Beijing. Compared with Beijing, the effect of energy conservation and carbon emissions reduction in Tianjin 
and Hebei are better. Strengthening work of energy conservation and carbon emissions reduction in Tianjin and Hebei is possible to achieve absolute reduction in carbon emissions.

The transportation output scale effect leads to sharp increase in carbon emissions, and it is the main factor to boost the growth of carbon emissions. It shows the transportation economic growth is still based on energy consumption. In terms of driving factor of energy intensity, energy intensity effect has led to the reduction of carbon emissions in the transport sector in Tianjin and Hebei. But inhibition effect has decreased in recent years. And the energy intensity effect has leads to the increase of transport carbon emissions in Beijing, it showsthe potential of energy saving and emission reduction is huge.In terms of the energy structure effect, the transportation energy structure effect of Beijing, Tianjin and Hebei have weak positive contributions or negative contributions to the overall of carbon emissions, and it suggests that energy structure of China's transportation industry is still relatively simple.

\section{Reference}

[1] Hoekstra R, Van den Bergh J C J M. Comparing structural decomposition analysis and index [J]. Energy economics, 2003, 25(1): 39-64.

[2] Tapio P. Towards a theory of decoupling: degrees of decoupling in the EU and the case of road traffic in Finland between 1970and 2001. Transport Policy, 2005. 12(2): 137-151.

[3] YA Qi, ZHU Rong-hui, ZHAO Xiao-qiang. Calculation decoupling analysis and scenario prediction of carbon emissions of transportation in China [J]. Journal of Chang'an University: Natural Science Edition, 2014, 05:77-83.

[4]MA Yue-yue, WANG Wei-guo. Character of carbon emission of logistics industry in China and its affecting factors decomposition analysis: based on LMDI technique [J]. Mathematics in Practice and Theory,2013,10:31-42. 